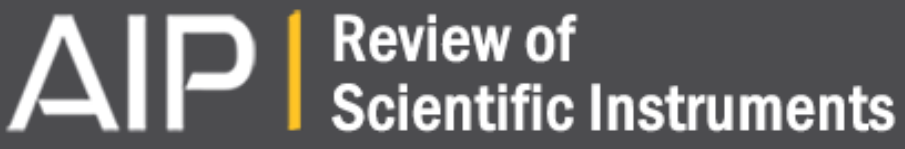

\section{Sub-Kelvin ac magnetic susceptometry}

M. A. Schmidt, D. M. Silevitch, N. Woo, and T. F. Rosenbaum

Citation: Review of Scientific Instruments 84, 013901 (2013); doi: 10.1063/1.4773231

View online: http://dx.doi.org/10.1063/1.4773231

View Table of Contents: http://scitation.aip.org/content/aip/journal/rsi/84/1?ver=pdfcov

Published by the AIP Publishing

Articles you may be interested in

Calorimetric method of ac loss measurement in a rotating magnetic field

Rev. Sci. Instrum. 81, 074702 (2010); 10.1063/1.3458003

Calibration of low-temperature ac susceptometers with a copper cylinder standard

Rev. Sci. Instrum. 81, 025104 (2010); 10.1063/1.3309779

Nonmagnetic indenter-type high-pressure cell for magnetic measurements

Rev. Sci. Instrum. 78, 023909 (2007); 10.1063/1.2459512

Magneto-optical apparatus to measure ac magnetic susceptibility

Rev. Sci. Instrum. 75, 2351 (2004); 10.1063/1.1765758

A moving coil ac magnetic susceptometer

Rev. Sci. Instrum. 69, 431 (1998); 10.1063/1.1148677

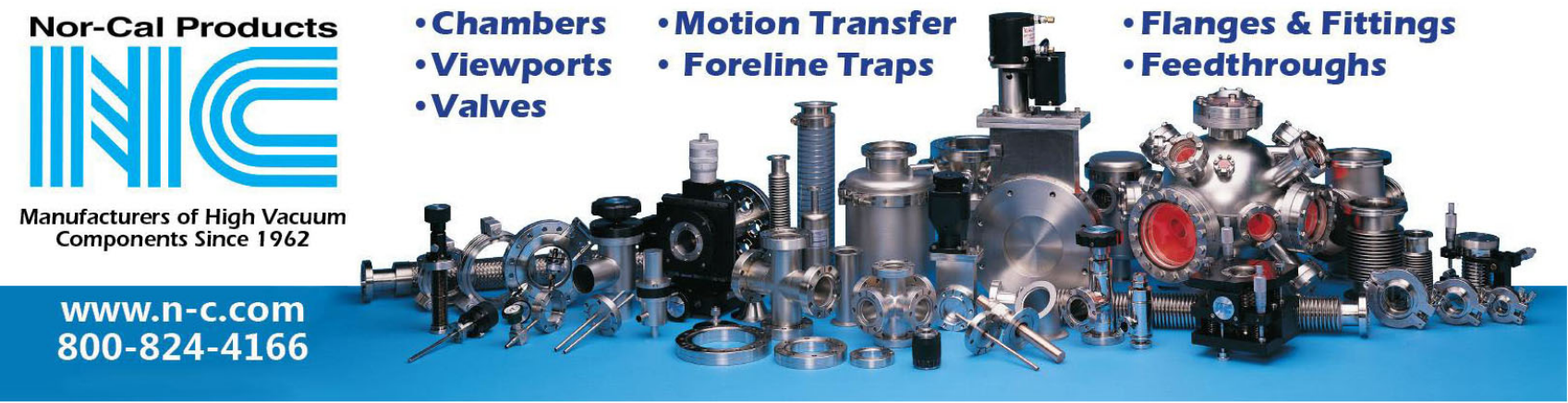




\title{
Sub-Kelvin ac magnetic susceptometry
}

\author{
M. A. Schmidt, D. M. Silevitch, N. Woo, and T. F. Rosenbaum ${ }^{\text {a) }}$ \\ The James Franck Institute and Department of Physics, The University of Chicago, \\ Chicago, Illinois 60637, USA
}

(Received 19 July 2012; accepted 9 December 2012; published online 2 January 2013)

\begin{abstract}
Designing an apparatus for experiments that operate deep into the milliKelvin regime requires a careful selection of materials and construction techniques, and often entails balancing mutually contradictory requirements. In the case of magnetic susceptibility measurements, this involves choosing materials that realize both high thermal conductivity for good heat sinking and low electrical conductivity for minimal eddy-current heating. A coil mounting system with a completely nonmetallic construction, achieved through the use of a sapphire skeleton and a machined carbon-fiber body, resolves this dilemma. This system permits the use of large ac magnetic field amplitudes at $\mathrm{kHz}$ frequencies and above, opening the door to studies of the nonlinear dynamics of a wide variety of magnetic systems that were previously inaccessible. (C) 2013 American Institute of Physics. [http://dx.doi.org/10.1063/1.4773231]
\end{abstract}

\section{INTRODUCTION}

Designing an experimental apparatus to function deep into the milliKelvin temperature range requires extensive and careful consideration of materials and construction techniques to optimize performance. Depending on the experiment, this can include considerations such as a high thermal conductivity at low temperatures to reduce thermal gradients, little or no paramagnetic or diamagnetic response, and electronic and nuclear spin configurations with minimal contributions to the specific heat. For most applications, high purity noble metals, in particular copper and silver, are suitable structural materials.

Experiments that require the application of ac magnetic fields present special challenges. The magnetic coils themselves can employ superconducting wire, eliminating resistive heating to reasonable fields, but the experimental cell and mount-for example, in an ac magnetic susceptibility measurement - cannot be superconducting because they fail to satisfy the requirements of flux penetration and high thermal conductivity. The most commonly used material in such circumstances, oxygen-free high-conductivity (OFHC) copper, has its own limitations. OFHC copper is chosen for its high thermal conductivity at satisfactory cost (compared to silver or gold) without a superconducting transition down to the lowest temperatures probed. However, the same electrons that produce the high thermal conductivity also provide a high electrical conductivity, which can result in significant induced eddy currents and consequent Ohmic heating. This limitation becomes acute at high frequencies and large drive fields, regions where many materials of both physical interest and technological import can reveal essential insights via their nonlinear response. In this article, we describe a mounting scheme for an ac magnetic susceptometer that largely eliminates the problems associated with eddy current heating by using an entirely non-metallic construction for the mount

a) Author to whom correspondence should be addressed. Electronic mail: tfr@uchicago.edu. and superconducting coils for the magnetic primaries. We characterize its thermal performance at milliKelvin temperatures with direct comparison to a conventional OFHC copper mount, and demonstrate the ability to probe the nonlinear susceptibility of a frustrated magnet at $\mathrm{kHz}$ frequencies.

\section{DESIGN AND CONSTRUCTION}

The basic design of an ac magnetic susceptometer consists of a set of nested, coaxial coils driven by an ac current passing through the outer "drive" coil. The resulting ac magnetic field induces a voltage on the inner "pickup" coil, which then can be sensed by a lock-in amplifier or other detection device. ${ }^{1,2}$ A sample sits inside the inner coil and its magnetic response contributes to the net flux through the pickup, altering the detected voltage. Susceptometers of this design are often wired in a gradiometer configuration, in which two identical coil sets are wired in series with opposite relative polarities to cancel the voltage response due to the mutual inductance of the coils themselves. ${ }^{1}$

The usual technique for mounting such a device in a cryostat is to clamp the coils in place with rings or a cylinder of a high thermal conductivity material such as OFHC copper, with the clamp then attached to the cold finger of the cryostat. This design approach optimizes the thermal conductivity between the susceptometer/sample assembly and the cryostat; however, the all-metallic construction presents limitations due to the effects of eddy current heating. The voltage induced inside the mount is given by $V=-\frac{d \Phi}{d t}$, so for an ac magnetic field of amplitude $\mathrm{H}$ and frequency $f$, the power dissipated inside the mount scales as

$$
P \propto \frac{(f H)^{2}}{\rho},
$$

where $\rho$ is the resistivity of the mount material. For a low resistivity material such as copper, fields of a few Oe at several $\mathrm{kHz}$ can result in tens or hundreds of microwatts of power dissipation, significant on the scale of the cooling power of a helium dilution refrigerator. This has the practical effect of 
limiting the amplitudes and frequencies that can be accessed by the instrument. Techniques such as cutting slots in the metallic clamp can mitigate heating, but not eliminate it.

In conventional linear ac magnetic susceptometry, the applied magnetic field consists of a single sine wave with frequency typically in the range of $0.1 \mathrm{~Hz}$ to $100 \mathrm{kHz}$. In the case where the low end of the frequency range is fast compared to the thermal response time of the cryostat, the timefluctuating power dissipation generated by induced currents will appear to be effectively constant. This can be partially mitigated by placing the control thermometer in close thermal proximity to the experimental cell and using a feedback loop to account for the $\mathrm{I}^{2} \mathrm{R}$ heating, as long as the cryostat has sufficient excess cooling power to absorb the load and thermal gradients are not disruptive. Nonlinear ac susceptibility, and in particular the technique of magnetic pump-probe spectroscopy, presents additional, more stringent constraints. In a pump-probe technique, ${ }^{3}$ two ac fields are applied simultaneously: a relatively large pump field at fixed frequency and a smaller probe field, which is swept through a range of frequencies around the pump frequency. Due to this superposition of two frequencies, the overall power dissipated exhibits a pair of beat signals at $\left(f_{\text {pump }}+f_{\text {probe }}\right) / 2$ and $\left|f_{\text {pump }}-f_{\text {probe }}\right|$. The former may be, as in the single frequency case, fast compared to the thermal timescales of the cryostat and hence can be addressed by standard temperature control techniques. The latter, however, can be small: on the order of $1-10 \mathrm{mHz}$ for experiments seeking to resolve the structure of tightly defined resonance modes. These frequencies correspond to periods of approximately 1 to $10 \mathrm{~min}$, resulting in an oscillatory heat load at a timescale comparable to the response time of a cryogenic temperature controller, giving rise to the possibility of ringing in the temperature feedback loop and significant variations in temperature about the nominal set point.

Since the power dissipated scales inversely with the resistivity of the mount material (Eq. (1)), at first glance it would appear to be advantageous to work with a material of higher resistivity. Unfortunately, the electrical and thermal resistivities of metals are tightly coupled. This means that choosing a metal with a high electrical resistivity will result in poor thermal conductivity and hence be unsuitable for use as a mounting material in a cryogenic environment. The Wiedemann-Franz law, however, only applies to materials where the primary mechanism of conductivity is the movement of free electrons. In materials where heat transport is phonon-dominated, high thermal conductivity can coexist with high electrical resistivity. Thus, the ideal material for mount construction would be an electrical insulator with a high density of phonon modes.

Rigid crystalline materials such as diamond and sapphire meet these criteria, with extremely high electrical resistivities at low temperature and a high density of phonon modes giving a relatively good thermal conductivity $\kappa / \mathrm{T}^{3} \sim 0.05 \mathrm{~W} / \mathrm{K}-\mathrm{cm}$ below $4 \mathrm{~K}^{4}$ (approximately, $10^{-3}$ of the thermal conductivity of OFHC copper at $\mathrm{T}=100 \mathrm{mK}$ ). Diamond may be ruled out due to the financial and availability constraints of single crystals of size sufficient to form into a coil mount. Synthetic sapphire, however, is readily available in plates and rods in a wide variety of sizes. Due to the hardness of the material and the complexity of the geometry of a coil clamping system (and consequent difficulty in machining and forming), it is not feasible to construct a pure sapphire mount. We therefore consider a heterogeneous design where a skeleton of sapphire rods and plates provides a suitable thermal conductivity and a more machinable insulating material is used to form the bulk of the mount body.

One class of materials that has recently become of interest for cryogenic applications is carbon-fiber composites. ${ }^{5-7}$ Carbon fiber reinforced polymers (CFRPs) are a family of strong, lightweight composite materials consisting of a polymer, typically epoxy, impregnated with woven layers of carbon fiber. The actual carbon fiber consists of bundles of several thousand carbon filaments, each only 5 to $10 \mu \mathrm{m}$ in diameter. ${ }^{8}$ The bundles are woven into a flexible fabric, which can be molded into myriad shapes. The most common commercially available shape is a simple plate, which can be obtained cheaply and easily from many suppliers. CFRPs, which henceforth we refer to simply as "carbon fiber," are used in a wide variety of commercial applications, from aerospace ${ }^{5}$ to high-end nautical, and bicycle manufacturing. Carbon fiber sheets can be bonded together readily using commercially available epoxies to build more complicated structures. Furthermore, carbon fiber composites can be machined to a precision of $0.025 \mathrm{~mm}$ (0.001 in.) with only moderate adjustments of the tooling, which makes them good candidates for a scientific apparatus.

In addition to their useful mechanical properties, carbon fiber has useful electrical and thermal properties at cryogenic temperatures. Owing to the planar nature of the carbon structure, carbon fiber composites display anisotropic thermal and electrical conductivities, typically an order of magnitude smaller in the plane of the carbon fibers than out of the plane. ${ }^{6}$ Carbon fiber composites have electrical resistivities at room temperature in the range of 1500 to $7400 \mu \Omega-\mathrm{cm},{ }^{6} \mathrm{com}-$ pared to the typical room temperature value of $1.6 \mu \Omega$-cm for OFHC copper. ${ }^{9}$ The electrical resistivity of carbon fiber degrades sharply at cryogenic temperatures, ${ }^{6}$ whereas the electrical resistivity of copper improves at low temperature. ${ }^{9}$ The thermal conductivity of carbon fiber also degrades at cryogenic temperatures, but it remains two to three orders of magnitude larger at $\mathrm{T}=1 \mathrm{~K}$ than other machinable, insulating materials such as nylon or Teflon. ${ }^{7,9}$

We have designed and manufactured a coil mount that consists almost exclusively of carbon fiber and sapphire, with small amounts of 3M DP-420 epoxy and GE 7031 varnish used to fasten certain elements. The main design feature is the skeleton of sapphire in which machined carbon fiber blocks sit to firmly engage the coils. The goal is to minimize the distance between the coils and the sapphire, using only enough carbon fiber to firmly grasp the coils and to hold the sapphire cage together, as shown in Fig. 1(a) and 1(b). Rather than using metal screws, we use bifurcated mortise and tenon joints, and pins to secure the various pieces together. With this design, the minimum distance between the coils and the sapphire rods is less than $2.54 \mathrm{~mm}$ ( $0.100 \mathrm{in}$.) on the sides, top, and bottom of the coils.

In order to construct these parts, we started with a $3.175 \mathrm{~mm}$ (0.125 in.) thick carbon fiber sheet, available from 

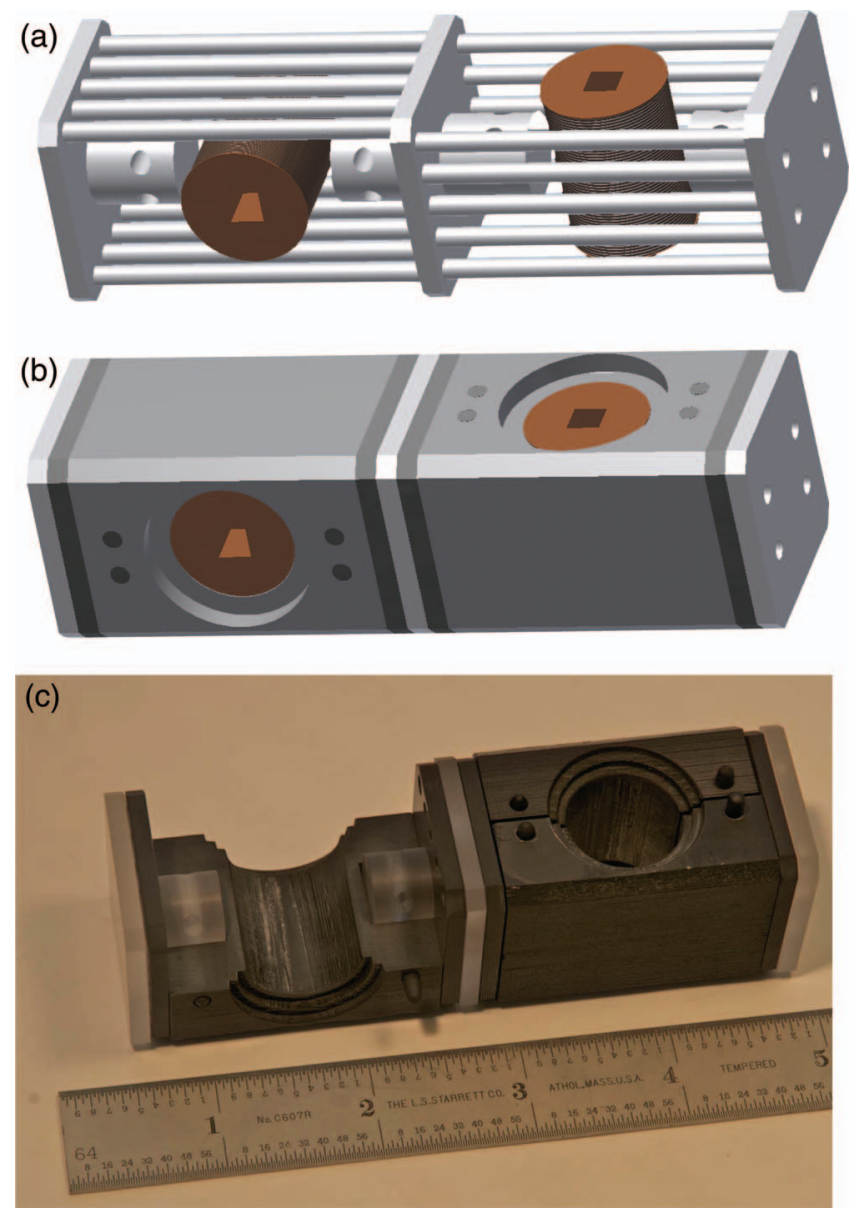

FIG. 1. Structure and construction of the sapphire/carbon-fiber susceptometer mount. Renderings of the mount design show (a) the sapphire plate and rod skeleton and (b) the complete structure including both sapphire and carbon-fiber components. (c) Photograph of the partially assembled mount, showing mortise and tenon design for fastening the carbon-fiber blocks to the sapphire skeleton. Pins machined from rods of carbon-fiber composite pass through holes in both the sapphire skeleton and the carbon-fiber blocks. GE 7031 varnish is used to hold these rods in position.

industrial distributors such as McMaster-Carr. We cut this sheet into $100 \mathrm{~mm} \times 50 \mathrm{~mm}$ strips, and after cleaning the surfaces with ethanol, we then applied 3M DP-420 epoxy to the faces of the strips and stacked them to form a solid block of material roughly $100 \mathrm{~mm} \times 50 \mathrm{~mm} \times 50 \mathrm{~mm}$ in size. We clamped the stack in a vice and let the epoxy cure $24+$ $\mathrm{h}$ to set fully and preclude delamination between layers in the stack during machining. Given that carbon fiber is very abrasive, carbide tooling must be used, and cuts must be made at slower speed than normally used for metals. Several additional safety precautions must be followed during machining: latex gloves must be worn when handling the cross-cut edges of the material to avoid splinters; a dust-mask must be worn; and a vacuum should be used at the point of the cut to limit the amount of residual carbon dust in the working area. If proper safety and technique protocols are followed, stacks of carbon fiber epoxy composites can be machined to very high precision, typically to $0.025 \mathrm{~mm}$ or better without significant risk of delamination or chipping, provided that the principle cuts are either parallel or perpendicular to the woven carbon fibers. Once the part is completed, we found it useful to apply a thin layer of epoxy to any exposed edges across layers of fiber. This helped prevent chipping and delamination. The surfaces of the material can be polished to a very uniform, smooth finish using wet, 1200-grit paper on a sanding block; this sanding also helps remove any stray splinters, and when combined with the application of epoxy to exposed edges, leads to a very desirable finish.

The sapphire parts were custom made by Insaco Corp, a supplier of sapphire and other hard crystalline materials. The parts were ground from bulk single crystals of approximate shape and size. Purchasing custom sapphire parts involves substantial expense, but can be minimized by restricting the shapes to simple geometries of rods and plates. The only additional assembly required was to varnish the tenons to the sapphire and carbon plates to form rigid structures that accept the $3.05 \mathrm{~mm}$ (0.120 in.), longitudinal sapphire rods; these composite plates with pin acceptors can be seen in the middle and on either end of the completed and partially assembled mount in Fig. 1(c).

\section{PERFORMANCE}

To test the performance of the sapphire/carbon-fiber coil mount, we have performed a series of pump/probe studies in a $400 \mu \mathrm{W}$ helium dilution refrigerator using a conventional OFHC copper coil mount as our control. In order to remove any heating effects due to direct Ohmic heating, we have used $\mathrm{Cu}$-clad superconducting $\mathrm{NbTi}$ wire in the primary coils, and we used the same set of coils for all measurements discussed here. We initially characterized the temperature of the mount on initial cooling from $2 \mathrm{~K}$ to $90 \mathrm{mK}$ and subsequent equilibration, followed by a series of small temperature increases. We then carried out all of the pump/probe experiments at the nominal mixing chamber temperature set point of $\mathrm{T}_{\mathrm{MC}}=0.090 \mathrm{~K}$, where the refrigerator mixing chamber heater power output is typically in the range $\mathrm{P}_{\mathrm{MC}}=[100200] \mu \mathrm{W}$, depending on the specific details of the pump and probe fields. In all studies, we measured the mixing chamber temperature and the temperature at the coil mount at $1 \mathrm{~s}$ intervals during the runs.

We show in the left panel of Fig. 2 the thermal behavior of the coil mount after initial cooling from high temperature to $90 \mathrm{mK}$. The temperature was measured using a calibrated $\mathrm{RuO}_{2}$ thermometer attached via GE7031 varnish to the bottommost plate of the coil mount, and thus represents a worst-case measure of the device performance. The system exhibits slow cooling characterized by a double-exponential time dependence with time constants of 4900 and $78000 \mathrm{~s}$. The shorter time constant corresponds to the superior thermal contact afforded by the sapphire rods, while the slow relaxation time is characteristic of many amorphous materials at sub-Kelvin temperatures with large heat capacities due to a population of two-level systems. ${ }^{10}$ The thermal behavior of the carbon fiber/epoxy matrix dominates during initial cooling and points to the need to allow the mount to thoroughly thermalize before acquiring data.

Once the mount has thermalized, the overall thermal behavior of the system can be determined by incrementing the nominal cryostat temperature by a small step and measuring 

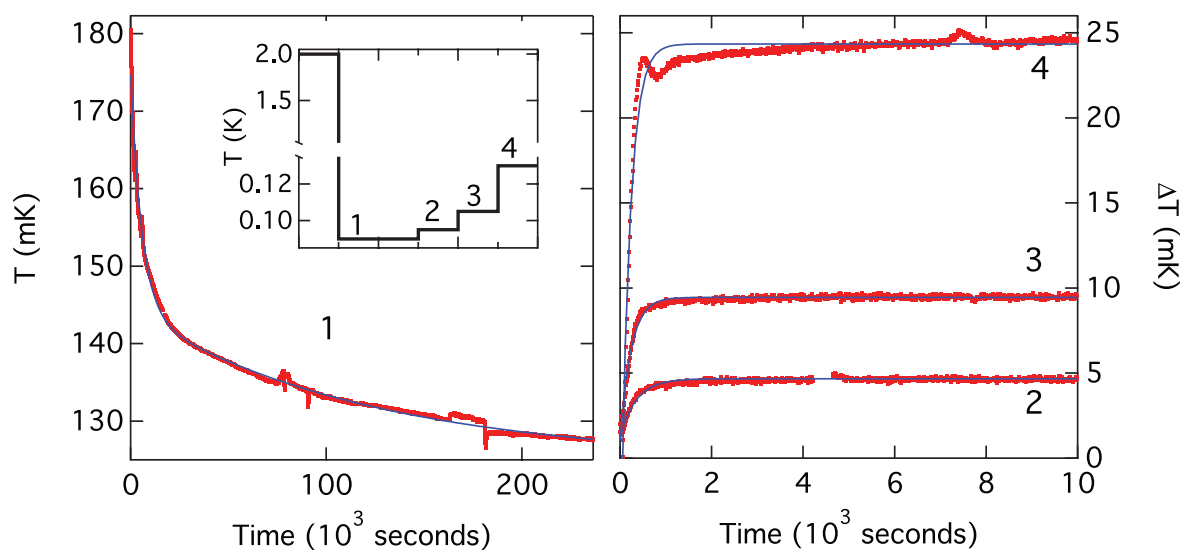

FIG. 2. Temperature as a function of time at the bottom plate of the sapphire/carbon-fiber mount. Left panel: Temperature vs. time after a quench from $2 \mathrm{~K}$ to $90 \mathrm{mK}$. Fit is a double exponential with time constants of 4900 and $78000 \mathrm{~s}$. Right panel: Temperature change vs. time after the mixing chamber temperature is changed by 5, 10, and $25 \mathrm{mK}$ (traces 2, 3, and 4, respectively). Fits are single exponentials with time constants of 290, 240, and 200 s, respectively. The oscillations in trace 4, the largest thermal step, are due to the proportional integral differential (PID) temperature control at the mixing chamber. Inset: Schematic showing mixing chamber temperature history vs. time.

the equilibration time. This procedure is illustrated in the right panel of Fig. 2 for temperature steps of 5, 10, and $25 \mathrm{mK}$. As can be seen from the compressed horizontal scale of this panel, the equilibration times are dramatically shorter than the initial cooling time, with the mount temperature following a single-exponential functional form with a time constant of 200 to $300 \mathrm{~s}$ depending on the size of the thermal increment. The contrast between these short equilibration times and the lengthy, initial thermalization period indicates that once the system has cooled, the thermal behavior is dominated by conduction through the sapphire rods rather than the behavior of the carbon fiber.

We compare in Fig. 3 the results of a pump/probe experiment with a frequency-varying $35 \mathrm{mOe}$ probe field and a fixed-frequency 525 mOe pump field at $19.959 \mathrm{~Hz}$ using both the sapphire/carbon-fiber and traditional OFHC copper mounts. The probe field varies between [19.954, 19.964] Hz, measured at $1 \mathrm{mHz}$ intervals. As expected, the copper mount demonstrates a large $(31 \mathrm{mK})$ temperature offset due to the fast oscillation, and a $5 \mathrm{mK}$ peak-to-peak oscillation in temperature during the course of the experiment. This oscillation occurs precisely at the beat frequency. The sapphire/carbonfiber mount performs markedly better under the same experimental conditions: the temperature offset from the mixing chamber has been reduced to $4 \mathrm{mK}$, with an oscillation of less than $1 \mathrm{mK}$. Interestingly, this oscillation at the same amplitude can be seen clearly in the mixing chamber temperature as well. This indicates that the residual heating is not due to the sapphire/carbon-fiber coil mount itself, but rather arises from eddy current heating of the OFHC copper used in the cold finger extending vertically downwards $22 \mathrm{~cm}$ from the mixing chamber into the bore of a dc superconducting magnet, where the sample and susceptometer are positioned (see inset to Fig. 4).

We display in Fig. 4 the results of a series of pump/probe experiments using $35 \mathrm{mOe}$ probe and $525 \mathrm{mOe}$ pump fields as before, but now varying the pump frequency over three decades: $f_{\text {pump }}=\{19.959,199.59,1995.9\}$ Hz. In each case, we varied the probe frequency in a $\pm 5 \mathrm{mHz}$ window around the pump field. As expected from Eq. (1), we find that as the pump frequency increases, the heating effects also increase; however, the temperature offset between the lowest and highest pump frequencies increases by only $\sim 15 \mathrm{mK}$ despite increasing the pump frequency by two orders of magnitude. Again, this heating is due chiefly to the copper cold finger. Spectroscopic and nonlinear studies are now possible in regimes previously made inaccessible by eddy current heating and thermal gradients.

As an explicit example of the new capabilities gained using this coil mount, we have performed a pump/probe experiment on a sample of $0.1 \%$ neodymium-doped gadolinium

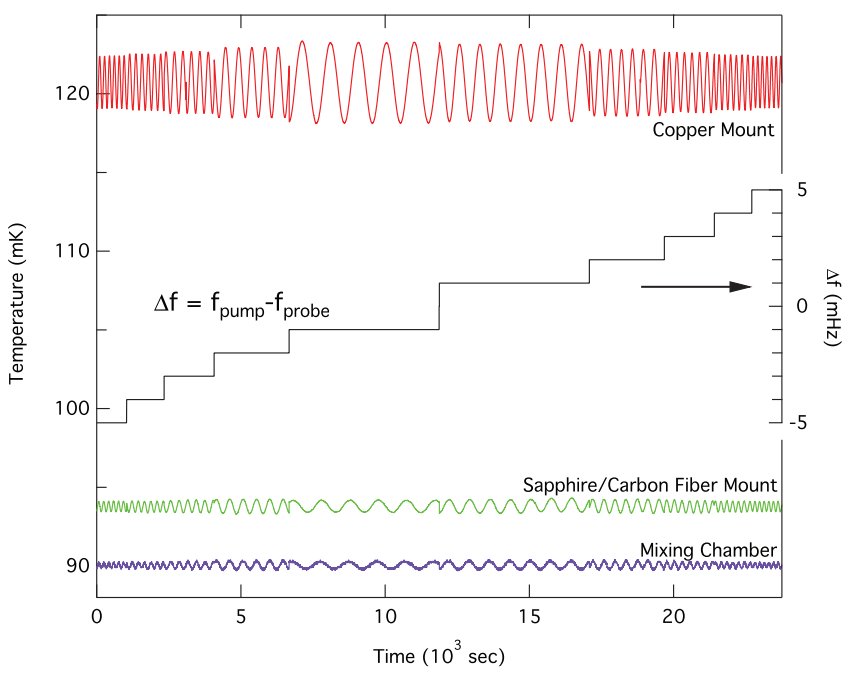

FIG. 3. Thermal performance of the sapphire/carbon-fiber mount compared to a conventional OFHC copper mount. The system is driven in a pumpprobe configuration with a $525 \mathrm{mOe} / 19.959 \mathrm{~Hz}$ pump field and a $35 \mathrm{mOe}$ probe field varied by $\pm 5 \mathrm{mHz}$ around the pump frequency. A PID control loop maintains a temperature of $90 \mathrm{mK}$ at the mixing chamber. The OFHC copper mount sees an average heating of $31 \mathrm{mK}$ and a beat signal at $\Delta f=\left|f_{\text {pump }}-f_{\text {probe }}\right|$ with peak-peak amplitude of $5 \mathrm{mK}$. Under the same drive field conditions, the sapphire/carbon-fiber mount saw a substantially improved $3.7 \mathrm{mK}$ average shift with a beat amplitude of $0.7 \mathrm{mK}$. The mixing chamber temperature, shown here for the measurement with the sapphire/carbon-fiber mount, exhibits a small oscillation around the $90 \mathrm{mK}$ set point due to the comparable timescales of $\Delta f$ and the PID control loop. 


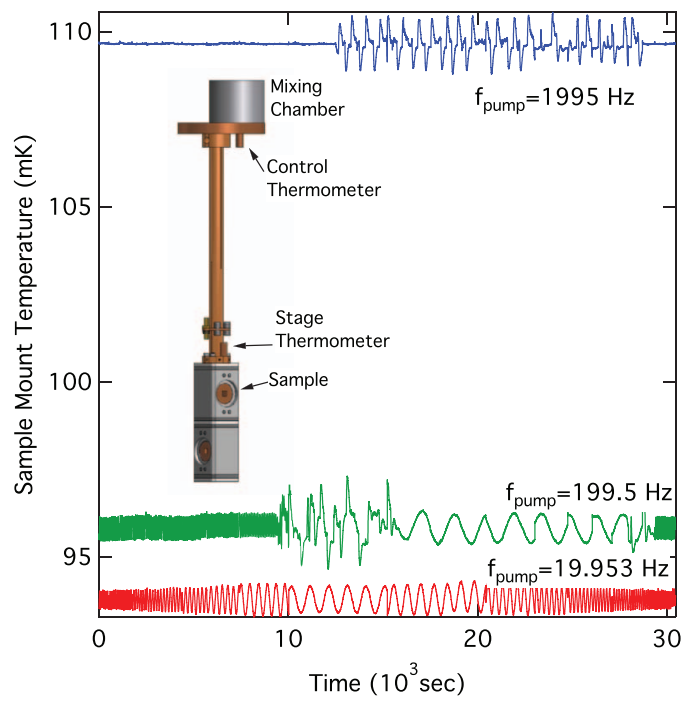

FIG. 4. Thermal performance for the sapphire/carbon-fiber mount in pump/probe spectroscopy measurements as a function of pump frequency. The 525 mOe pump is varied by two orders of magnitude from $20 \mathrm{~Hz}$ to $2 \mathrm{kHz}$ while the mixing chamber temperature was maintained at $90 \mathrm{mK}$. At the highest pump frequency, the average thermal shift due to the pump remains below $20 \mathrm{mK}$. Pump-probe measurements at these frequencies are impossible with a copper mount, as the substantial heat loss results in an immediate loss of temperature control and rapid warming of the system by at least $300 \mathrm{mK}$. The inset diagrams the thermometry. $\mathrm{A} \mathrm{RuO}_{2}$ control thermometer mounted on the mixing chamber is used as input for a PID control loop that drives a heater on the mixing chamber, while a germanium thermometer mounted at the base of the cold finger records the sample temperature.

gallium garnet (Nd:GGG) at $1995.9 \mathrm{~Hz}$ using a $700 \mathrm{mOe}$ amplitude pump field. Studies on the pure form of this material ${ }^{11}$ have shown that geometric frustration in the magnetic unit cell gives rise to weakly coupled clusters of coherent spins. Using the magnetic pump/probe technique, it is possible to

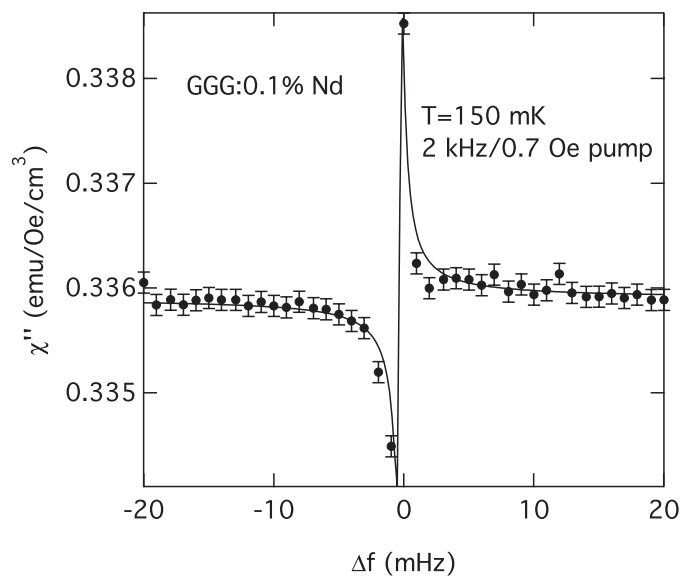

FIG. 5. Results of a pump/probe experiment on Nd-doped Gadolinium Gallium Garnet at $150 \mathrm{mK}$ using a $700 \mathrm{mOe}$ pump at $1995 \mathrm{~Hz}$. The imaginary part of the magnetic susceptibility, $\chi$, is plotted versus the difference between the pump and probe frequencies. The imaginary part is fit to a Fano resonance, ${ }^{12} \chi^{\prime} \propto \frac{\left(\frac{q \Gamma}{2}+\Delta f\right)^{2}}{\left(\Delta f^{2}+\left(\frac{\Gamma}{2}\right)^{2}\right)}$, about the pump frequency, indicating competition between relaxation pathways from the isolated magnetic clusters and the spin bath. address and manipulate these clusters while only weakly affecting the underlying Heisenberg spin background. Due to the problems with sample heating discussed in Sec. I, previous studies of the nonlinear properties of the material were limited to low frequencies (tens of $\mathrm{Hz}$ ). Using the new coilmount design presented here, we have been able to excite a Fano resonance (Fig. 5) in the imaginary part of the spectrum at a frequency nearly two orders of magnitude higher than previously possible. The presence of this feature in the nonlinear spectrum is an unmistakable indicator of interference between discrete and continuous relaxation pathways for the magnetic degrees of freedom, and it provides strong evidence for the existence of coherent spin clusters ${ }^{3}$ at length scales previously shielded from inquiry. These results on Nd-GGG in the challenging pump/probe configuration are interesting in and of themselves but most importantly serve as proof of principle for a coil-mount design that can open up entirely new regions of study of magnetic materials.

\section{CONCLUSIONS}

We have presented a design for a cryogenic coil mount that enables ac magnetic susceptibility experiments over a broad range of excitation amplitudes and frequencies down to milliKelvin temperatures. The key to the design is a combination of carbon fiber and custom sapphire parts that eliminates eddy-current heating in the coil mount and thermally outperforms a traditional OFHC copper coil mount. The performance could be improved even further by optimizing the aspect ratio of the coils themselves to decrease the field at the OFHC copper cold finger. A pump/probe experiment on a frustrated magnet illustrates the power of investigating the high-frequency, high-amplitude nonlinear response.

\section{ACKNOWLEDGMENTS}

We are indebted to $\mathrm{H}$. Krebs for his valuable guidance in machining the carbon fiber parts. This work was supported by the U.S. DOE Basic Energy Sciences (Grant No. DE-FG0299ER45789).

${ }^{1}$ L. J. M. Van De Klundert, C. De Rooij, M. Caspari, and L. C. Van Der Marel, Cryogenics 15, 577-589 (1975).

${ }^{2}$ W. L. Pillinger, P. S. Jastram, and J. G. Daunt, Rev. Sci. Instrum. 29, 159 (1958).

${ }^{3}$ D. M. Silevitch, C. M. S. Gannarelli, A. J. Fisher, G. Aeppli, and T. F. Rosenbaum, Phys. Rev. Lett. 99, 057203 (2007).

${ }^{4}$ M. W. Wolfmeyer and J. R. Dillinger, Phys. Lett. A 34, 247 (1971).

${ }^{5}$ A. T. Nettles and E. J. Bliss, "Low temperature mechanical testing of carbon-fiber/epoxy-resin composite materials," NASA Technical Paper 3663, 1996.

${ }^{6}$ J. K Park and T. J Kang, Carbon 40, 2125 (2002).

${ }^{7}$ C. I. Nicholls and H. M. Rosenberg, Cryogenics 24, 355-358 (1984).

${ }^{8}$ T. Horiuchi and T. Ooi, Cryogenics 35, 677-679 (1995).

${ }^{9}$ F. Pobell, Matter and Methods at Low Temperatures (Springer, Berlin, 1996).

${ }^{10}$ R. C. Zeller and R. O. Pohl, Phys. Rev. B 4, 2029-2041 (1971).

${ }^{11}$ S. Ghosh, T. F. Rosenbaum, and G. Aeppli, Phys. Rev. Lett. 101, 157205 (2008).

${ }^{12}$ U. Fano, Phys. Rev. 124, 1866 (1961). 